NASA Technical Memorandum 102324

\title{
Probabilistic Structural Analysis of Aerospace Components Using NESSUS
}

Michael C. Shiao and Vinod K. Nagpal

Sverdrup Technology, Inc.

NASA Lewis Research Center Group

Cleveland, Ohio

and

Christos C. Chamis

National Aeronautics and Space Administration

Lewis Research Center

Cleveland, Ohio

Presented at the

29th Structures, Structural Dynamics and Materials Conference cosponsored by the AIAA, ASME, ASCE, AHS, and ACS Williamsburg, Virginia, April 18-20, 1988

\section{NASA}




\title{
ORIGIMAS SMTTALAS \\ GOLOR HLUSTRATIONS
}

\section{PROBABILISTIC STRUCTURAL ANALYSIS OF AEROSPACE COMPONENTS USING NESSUS}

\author{
Michael C. Shiao* and Vinod K. Nagpal ${ }^{\dagger}$ \\ Sverdrup Technology, Inc. \\ NASA Lewis Research Center Group \\ Cleveland, Ohio 44135 \\ and \\ Christos C. Chamis ${ }^{\ddagger}$ \\ National Aeronautics and Space Administration \\ Lewis Research Center \\ Cleveland, Ohio 44135
}

\section{Summary}

Probabilistic structural analysis of a space shuttle main engine (SSME) turbopump blade is performed by using the computer code NESSUS (Numerical Evaluation of Stochastic Structures Under Stress). The goal of the analysis is to derive probabilistic characteristics of blade response for given probabilistic descriptions of uncertainties in blade geometry, material properties, temperature, and pressure distributions. Probability densities are calculated for critical blade responses. Risk assessment is performed assuming different failure models.

\section{Introduction}

Probabilistic structural analysis assesses the effects of uncertain or random input parameters on structural response. Unlike the traditional deterministic analysis, in which geometry, material properties, and loading are assumed known, probabilistic structural analysis treats these inputs as samples from probability distributions. This approach makes it possible to assess the effects of fluctuating loads, variable material properties and uncertain geometry on structural response. In the deterministic approach, these uncertainties are not quantified and are acknowledged by an empirical safety

\footnotetext{
- Research Engineer. tDepartment Director.

‡Senior Aerospace Scientist, Structures Division.
}

factor. Probabilistic structural analysis provides a more realistic and systematic way to evaluate structural performance and durability.

The NESSUS computer code (ref. 1), developed under the research program, Probabilistic Structural Analysis Methods for Select Space Propulsion System Components (PSAM) (ref. 2), combines finite element methods with probabilistic load and geometry input descriptions and structural reliability algorithms to provide an integrated approach to probabilistic structural analysis.

\section{Structure of NESSUS}

NESSUS consists of the following three major modules: NESSUS/PRE, NESSUS/FEM, and NESSUS/FPI.

NESSUS/PRE is a preprocessor used for the preparation of the statistical input needed to perform the probabilistic finite element analysis. It allows the user to describe a spatial domain defined by a set of discrete points, typically corresponding to the nodal points of a finite element mesh. One or more random fields may then be specified over this spatial domain by defining the mean value and standard deviation of the random variable at each point, together with an appropriate form of correlation. Correlated random variables are decomposed into a set of uncorrelated vectors by an eigenvalue transformation procedure. For strong correlation problems, the number of dominant random variables in the set of uncorrelated vectors will be much less than that of weakly correlated random variables. The computational time required for the analysis 


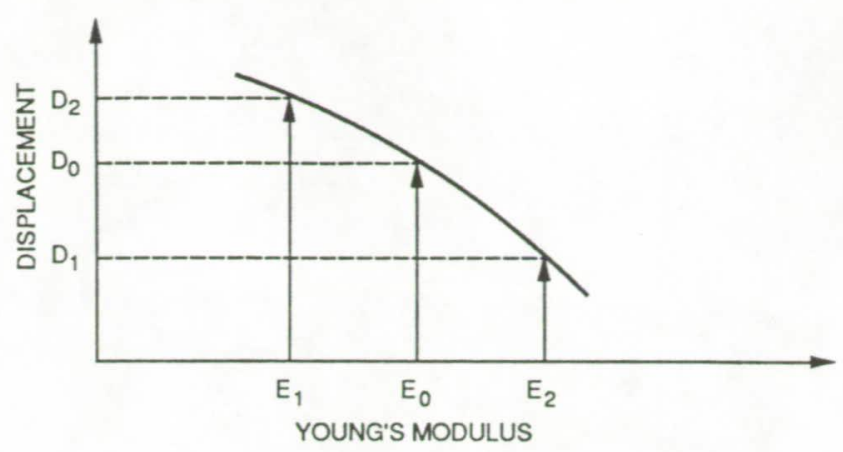

Figure 1.-Response function.

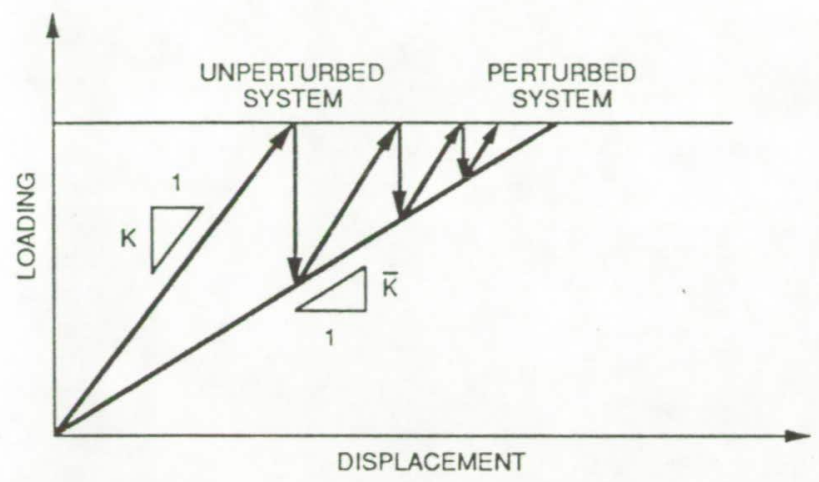

Figure 2.-Modified Newton iteration.

will also decrease due to the reduction of the number of uncorrelated random variables.

NESSUS/FEM is a finite element code used for structural analysis and parameter sensitivity evaluation. It generates a database containing all the response information corresponding to a small variation of each independent random variable. The algorithm used in NESSUS/FPI requires an explicit response function in terms of uncorrelated random variable in order to perform a reliability analysis. In complicated structural analysis problems, response can only be available implicitly through a finite element model. To overcome this difficulty, the response function is estimated numerically. As an example in figure 1, the response (dependent variable) to be considered is the displacement $D$ and the uncertainty involved is the modulus $E$ (independent variable). NESSUS/FEM calculates the displacements $D_{0}, D_{1}$, and $D_{2}$ corresponding to the modulus at $E_{0}, E_{1}, E_{2}$. Displacements $D_{1}$ and $D_{2}$ are computed by a method akin to the modified Newton nonlinear algorithm illustrated in figure 2 .

NESSUS/FPI implements an advanced reliability method known as the Fast Probability Integrator (refs. 3 and 4). This module extracts data from the database generated by NESSUS/FEM to develop a response or performance function in terms of uncorrelated random variables. The probabilistic structural response is calculated from the performance model. For a given response value, the probability of exceedence at this value is estimated by a reliability method, which treats the problem as a constrained minimization. This step is called a point probability estimate. The cumulative distribution function can be obtained numerically by running FPI at several response values. One alternative for generating the distribution function for any given response is to conduct a Monte Carlo simulation study. However, in general, direct simulation is very costly. NESSUS/FPI provides a method which not only produces an accurate probability distribution, but also requires less computing time than Monte Carlo simulation, especially in the low probability region.

\section{Concept of Probabilistic Structural Analysis by NESSUS}

Defining the loading, material properties, geometry, and boundary conditions as random variables, the probabilistic structural analysis is then performed with NESSUS. The structure can be considered as a filter to those random variables. The response variables, which can be displacements, strains and stresses at one or several locations, or natural frequencies, are the output of the filter and will be described by a cumulative distribution function (CDF) or a probability density function (PDF). The concept is illustrated in figure 3 .

\section{Turbine Blade Study by NESSUS}

Probabilistic structural analysis requires that the uncertainties involved be recognized and quantified. An SSME turbopump blade as shown in figure 4 is subjected to complex mechanical

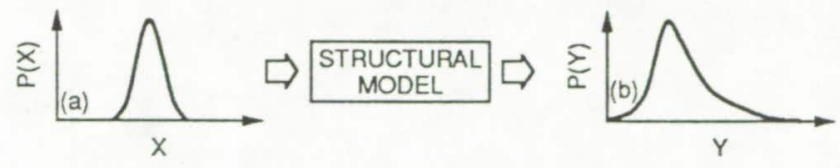

(a) Input PDF (load, material, geometry).

(b) Output PDF (displacement, strain, stress).

Figure 3.-Concept of probabilistic structural analysis.

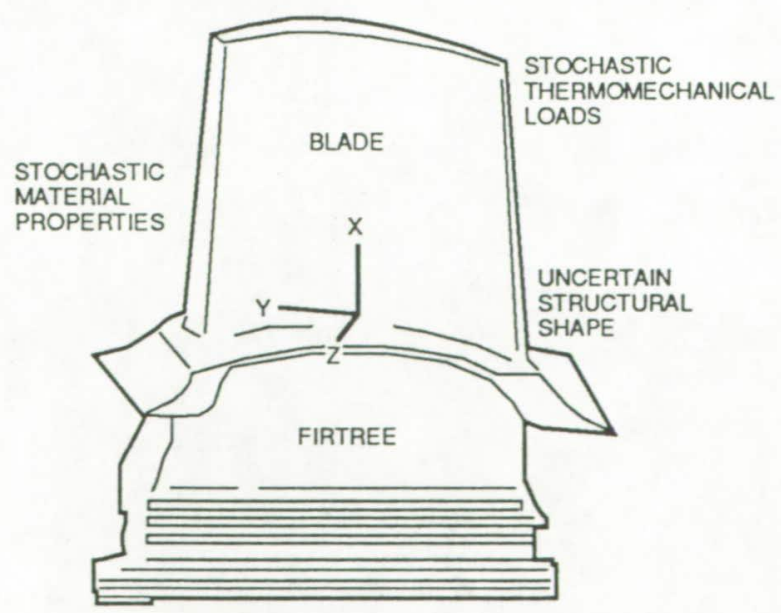

Figure 4.-A typical turbine blade. 
and thermal loads (ref. 5). The dominant loads on the turbine blade are centrifugal force, thermal load, and the differential pressure across the airfoil. Centrifugal force is induced by the blade rotation. Since it is difficult to maintain constant rotational speed, the centrifugal force is defined as a random variable. Random thermal load is due to combustion irregularities that cause a random temperature distribution in the blade. Differential pressure is also random because of pressure fluctuations. Uncertainties in the blade geometry exist as a result of the manufacturing process. The stochastic material properties are caused by nonuniformities in the material resulting from the fabrication. Uncertainty in the temperature distribution causes an additional level of material property uncertainty.

An SSME turbopump blade is modelled by 40 four-node shell elements with $\mathbf{5 5}$ nodal points. In this study, seven random fields are considered as listed in table I. Random variables for coordinate and temperature are defined at each grid point. Those for modulus and lateral pressure are defined at the element level. The mean values of coordinates random variables are the same as the deterministic coordinates. Mean temperature and pressure are shown in figures 5 and 6 . It is

TABLE I.-RANDOM INPUT DATA

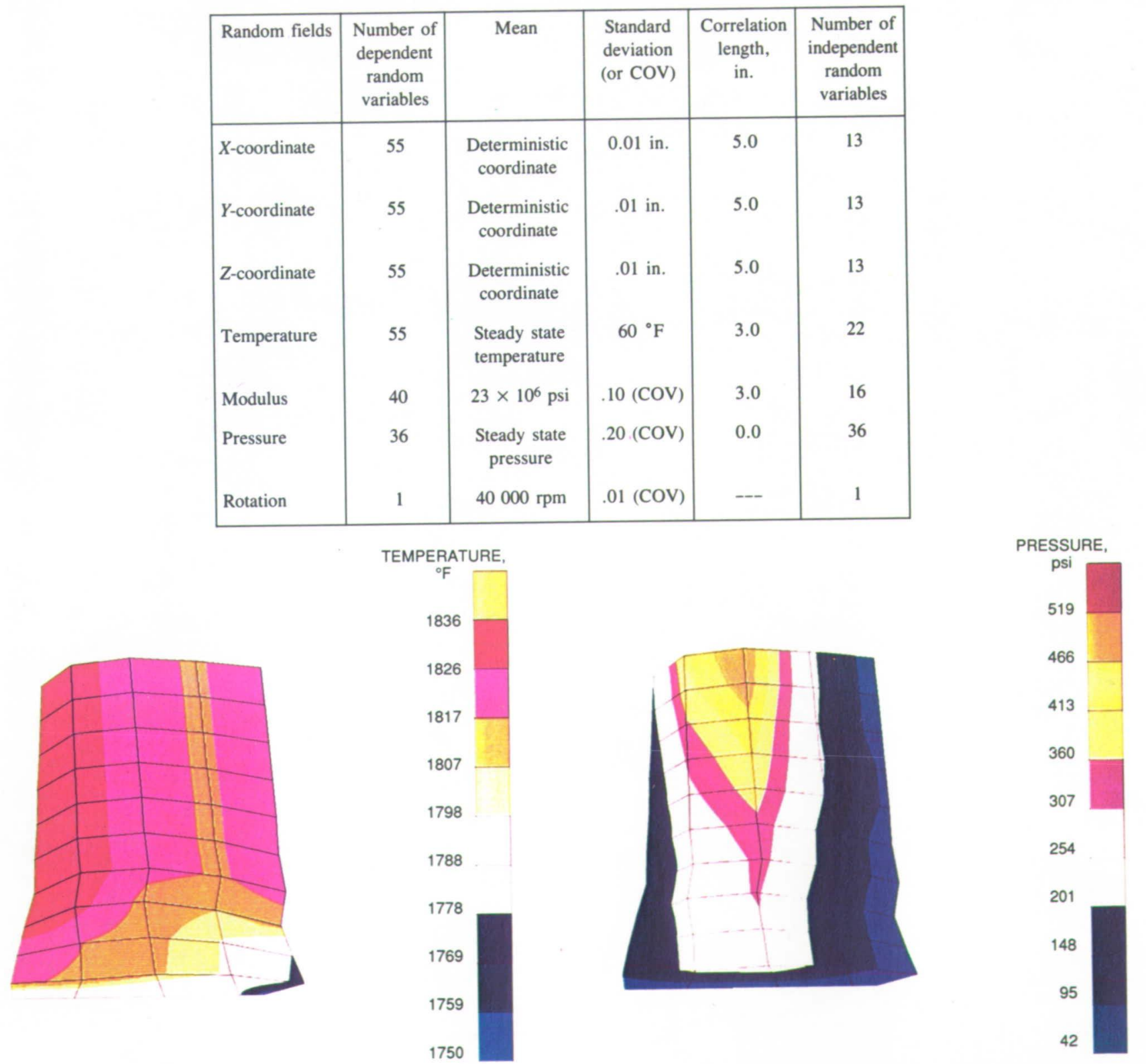

Figure 5.-Steady state temperature distribution.

Figure 6.-Steady state pressure distribution. 
assumed that the random fields are independent of each other. However, the correlation coefficients between random variables in the same field are defined as

$$
\rho\left(X_{i}, X_{j}\right)=\exp \left(-\Delta X_{i j} / S_{c}\right)
$$

where $X_{i}$ and $X_{j}$ are the $i$ th and $j$ th random variables in the $X$-coordinate random field; $\Delta X_{i j}$ is the distance between $X_{i}$ and $X_{j} . S_{c}$ is the correlation length. The larger the correlation length, the greater the correlation between the random variables in random field. When $S_{c}$ is extremely large, the random field can be represented by a single random variable. When $S_{c}$ approaches zero, the random variables are uncorrelated. In each correlated random field, the dependent random variables are functions of independent random variables through a decomposition procedure. The blade responses being analyzed are the effective stresses on both pressure and suction sides and displacements in the $X$ - and $Z$ directions. The mean, standard deviation, and coefficient of variation of effective stress on the pressure side are shown on figures 7(a) to (c). The largest mean effective stress is $102 \mathrm{ksi}$ at the lower corner of the leading edge. The coefficient

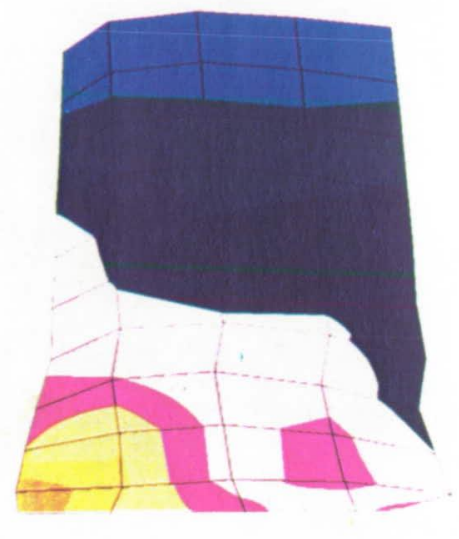

(a)

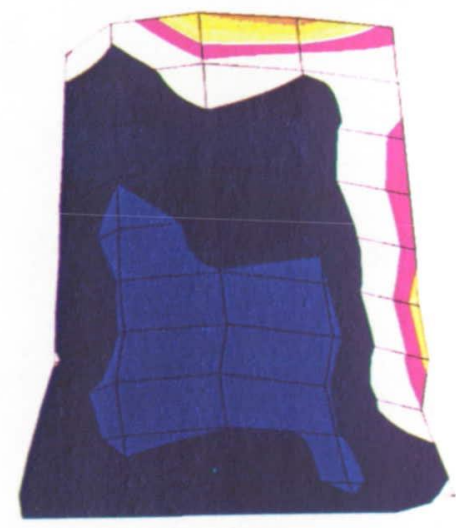

(c)
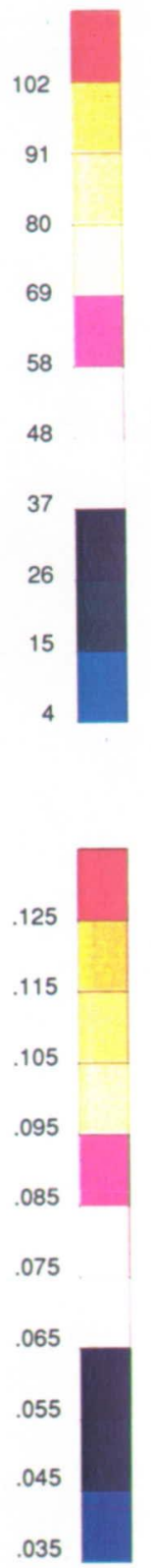

(a) Mean (pounds per square inch).

(c) Coefficient of variation.

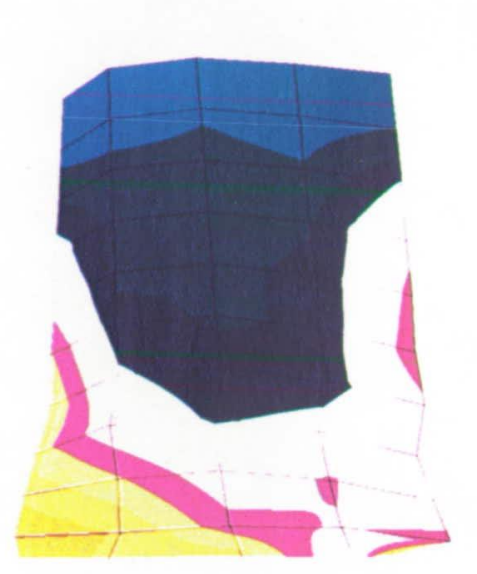

(b)
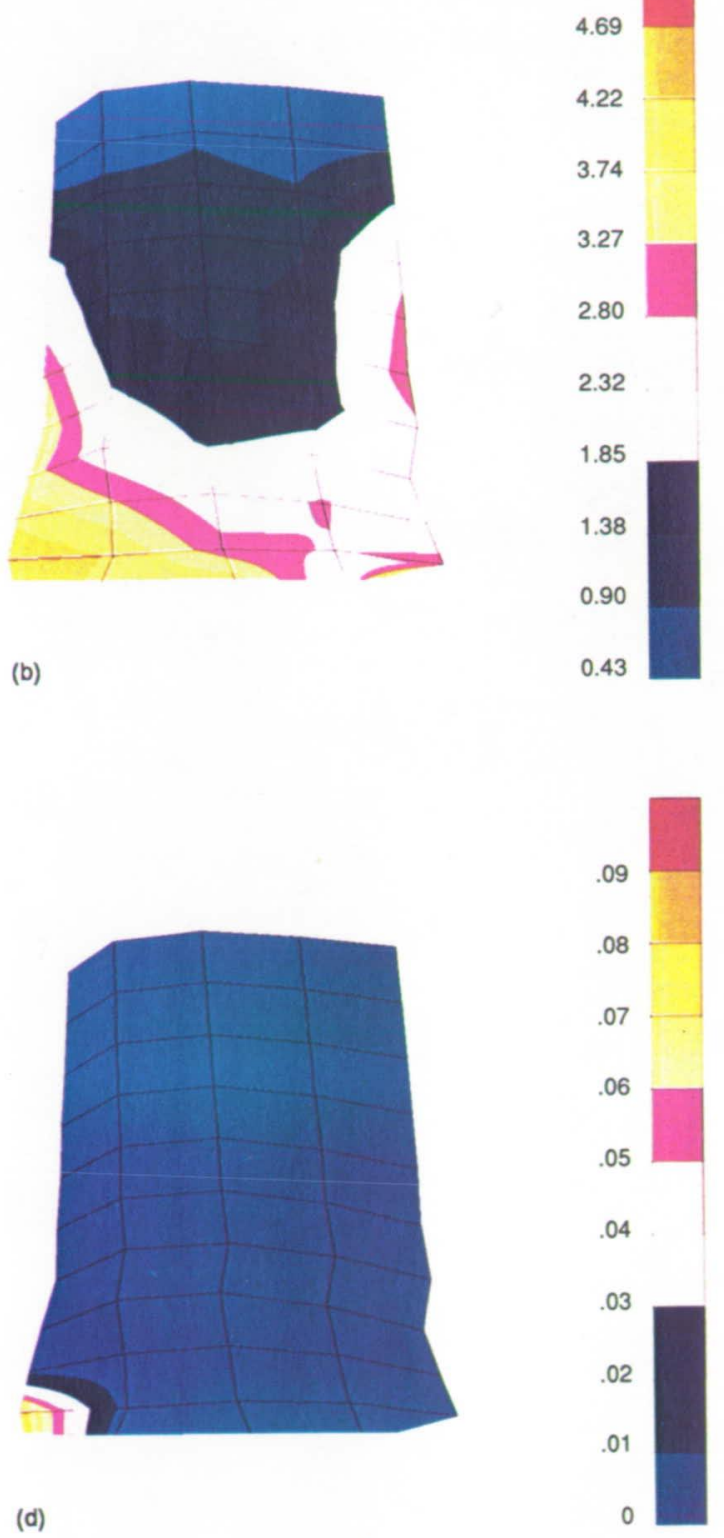

(b) Standard deviation (pounds per square inch).

(d) Probability of failure.

Figure 7.-Effective stress on pressure side. 
of variation associated with this critical stress is 3 percent. Based on this information, a blade risk analysis is performed. The failure mode is defined as the event where stress is greater than material strength. The probability of failure is calculated by

$$
P_{f}(i)=\int f_{S_{i}}(X) \quad F_{R_{i}}(X) d X
$$

where $f_{S_{i}}$ is the probability density function of effective stress at the $i$ th node computed by NESSUS/FPI. $F_{R_{i}}$ is the cumulative distribution function of strength at the $i$ th node, which is normally distributed with mean equal to $120 \mathrm{ksi}$ and standard deviation equal to $12 \mathrm{ksi}$. The failure probability at each nodal point is obtained and shown in figure 7(d). As expected, the location of highest failure probability is the lower left corner (blade root, leading edge). A similar study was performed for the effective stress on the suction side as shown in figure 8. Since the stress is much lower on the suction surface than that on the pressure surface, the failure probability is not as critical. For structures like blades, the displacements must be tightly controlled. The maximum deflection cannot exceed its allowable magnitude for clearance considerations. The mean and standard deviation of blade displacement in $\mathrm{X}$ -

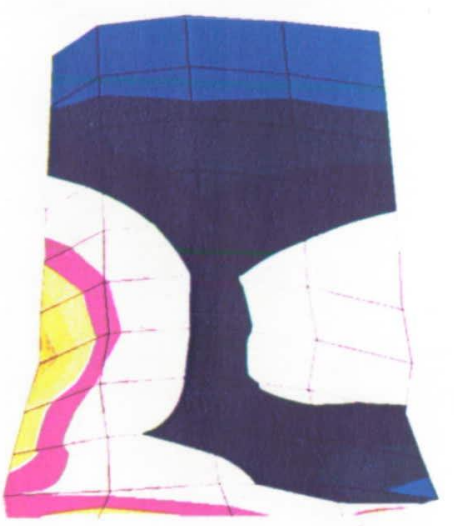

(a)
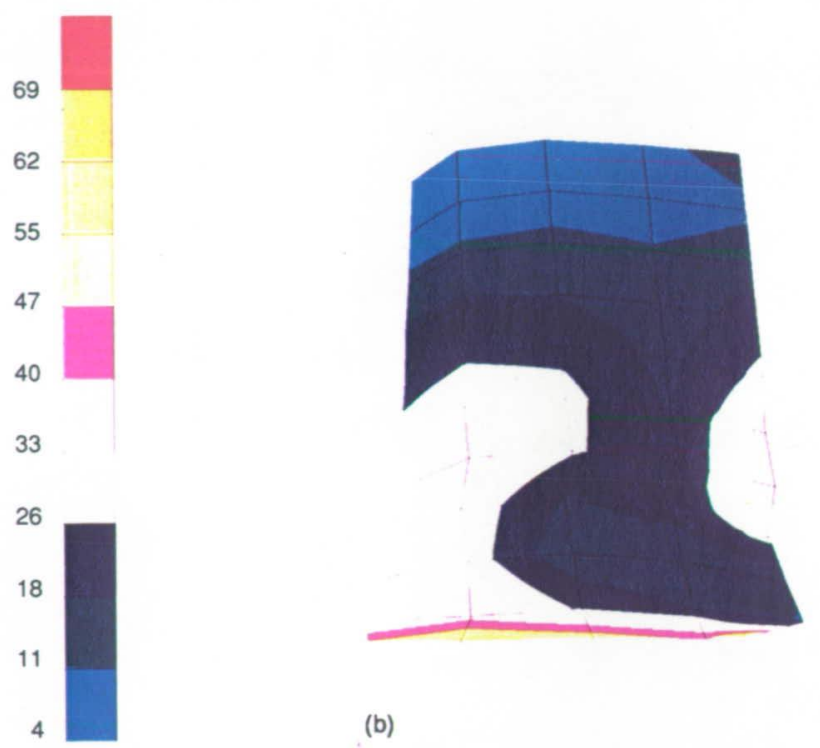

5.14

4.62

4.10

3.58

3.07

2.55

2.03

1.51

0.99

(b)

0.47

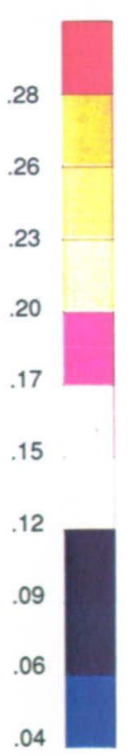

(a) Mean (pounds per square inch).

(c) Coefficient of variation.

(b) Standard deviation (pounds per square inch).

(d) Probability of failure.

Figure 8.-Effective stress on suction side. 
and Z-directions are shown in figures 9 and 10. Thus, it can be decided whether the deflection control is acceptable or not.

NESSUS/FPI also computes the sensitivity of the probability distribution for any response to the parameters of the independent random variables. This information, quantified by sensitivity factors, can be used to define the roles of the random variables in the reliability analysis. Sensitivity factors indicate which random variables are crucial and require more attention during the manufacturing process and in the environmental (loading, temperature) conditions. The sensitivity factors for the effective stress at the airfoil leading edge root are obtained. The five most influential random variables are listed in table II.
As expected, the temperature random field has insignificant effect on the stress. The $Z$-coordinate random field is a crucial one. The rotational speed which induces centrifugal forces is another important random variable. The uncertainty of the effective stress at root will be minimized significantly if the uncertainties of those five random variables are reduced. This information leads the designer to improve the design and reliability of the blade in a most efficient way. A similar analysis has been done for tip displacement in the $X$-direction. The five most influential random variables are shown in table III. In this case, the temperature has a dominant effect. The modulus should also be included in the reliability model.

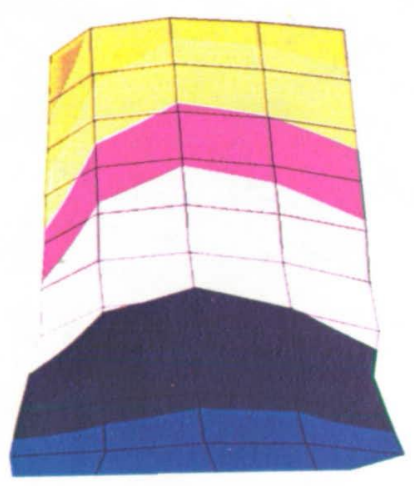

(a)

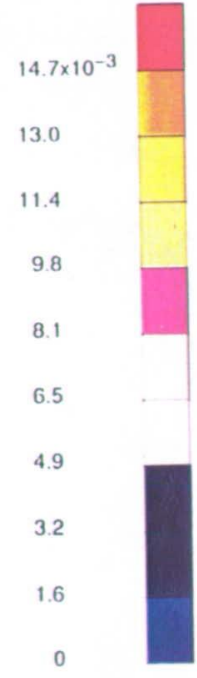

(a) Mean.

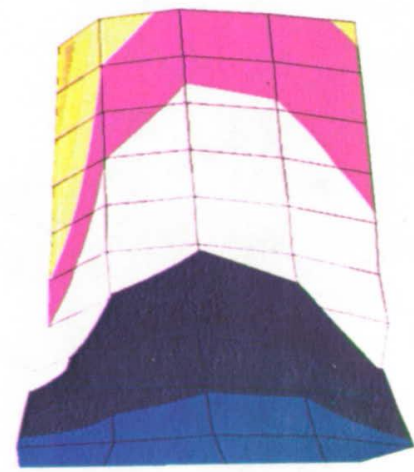

(b)

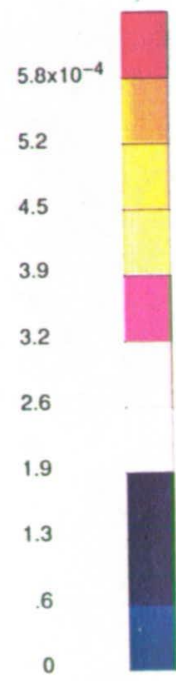

(b) Standard deviation

Figure 9.-Displacement in $X$-direction (inches). 


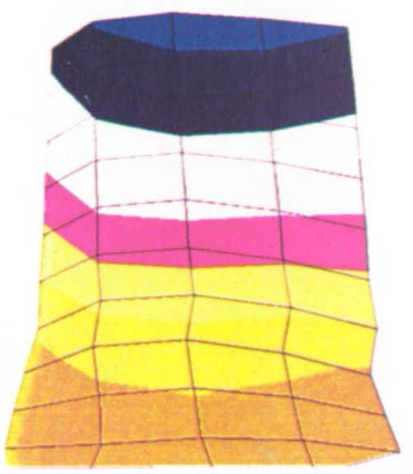

(a)

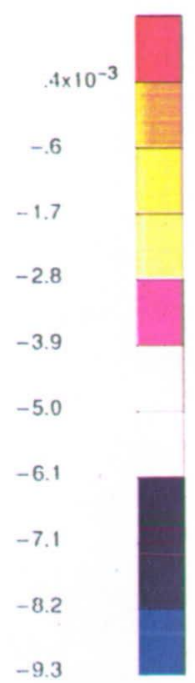

(a) Mean.

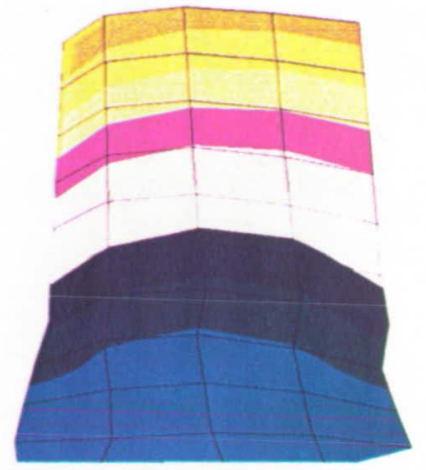

(b)

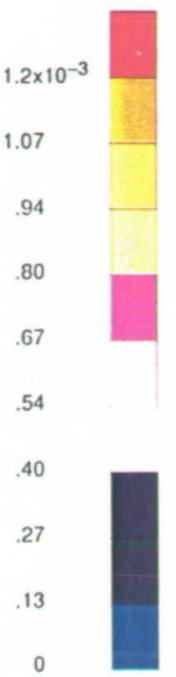

(b) Standard deviation.

Figure 10.-Displacement in Z-direction (inches).

TABLE II.-FIVE MOST INFLUENTIAL INDEPENDENT RANDOM VARIABLES TO THE EFFECTIVE STRESS AT ROOT

\begin{tabular}{|l|c|c|c|}
\hline Random fields & $\begin{array}{c}n \text {th Independent } \\
\text { random variable } \\
\text { in each field }\end{array}$ & $\begin{array}{c}\text { Sensitivity } \\
\text { factor }\end{array}$ & Ranking \\
\hline Z-coordinate & 1 & 0.204 & 4 \\
Z-coordinate & 2 & .610 & 1 \\
Z-coordinate & 5 & .273 & 3 \\
Modulus & 12 & .188 & 5 \\
Rotation speed & 1 & .326 & 2 \\
\hline
\end{tabular}

TABLE III.-FIVE MOST INFLUENTIAL INDEPENDENT RANDOM VARIABLES TO THE TIP DISPLACEMENT (X-COMPONENT)

\begin{tabular}{|l|c|c|c|}
\hline Random fields & $\begin{array}{c}n \text {th Independent } \\
\text { random variable } \\
\text { in each field }\end{array}$ & $\begin{array}{c}\text { Sensitivity } \\
\text { factor }\end{array}$ & Ranking \\
\hline Z-coordinate & 2 & 0.160 & 4 \\
Z-coordinate & 2 & .145 & 5 \\
Temperature & 1 & .855 & 1 \\
Temperature & 3 & .223 & 3 \\
Modulus & 1 & .345 & 2 \\
\hline
\end{tabular}

\section{Conclusion}

A space shuttle main engine (SSME) turbopump blade is analyzed probabilistically by using NESSUS (Numerical Evaluation of Stochastic Structures Under Stress) to account for the uncertainties in the blade geometry, material properties, temperature, and loading conditions. Probability distributions of structural responses such as displacements and stresses are obtained. Risk assessment is performed based on different failure criteria with known probability distributions of response variables. Sensitivity of any response to each random variable is also calculated. A redesign is necessary when the blade does not meet the safety requirements. Sensitivity factors provide a guide to modify the design in an efficient way for the reliability improvement.

\section{Lewis Research Center}

National Aeronautics and Space Administration Cleveland, Ohio, November 14, 1989 


\section{References}

1. "Probabilistic Structural Analysis Methods for Select Propulsion System Structural Components (PSAM)," Vols. 1-3, NASA CR-185125-VOL-1,-2,-3, 1989.

2. Chamis, C.C., "Probabilistic Structural Analysis Methods for Space Propulsion System Components," Probabilistic Engineering Mechanics, Vol. 2, June 1987, pp. 100-110 (also, NASA TM-88861).

3. Lind, N.C., Krenk, D., and Madsen, H.O., Methods of Structural Safety, Prentice-Hall, New York, 1985.

4. Wu, Y.T., "Demonstration of a New, Fast Probability Integration Method for Reliability Analysis," Advances in Aerospace Structural Analysis, O.H. Burnside and C.H. Parr, eds., ASME, New York, 1985, pp. 63-73.

5. Newell, J.F., Kurth, R.E., and Ho, H., "'Composite Load Spectra for Select Space Propulsion Structural Components," RI/RD86-123 AR-1, Rockwell International Corp., Canoga Park, CA, 1987, NASA CR-179496. 


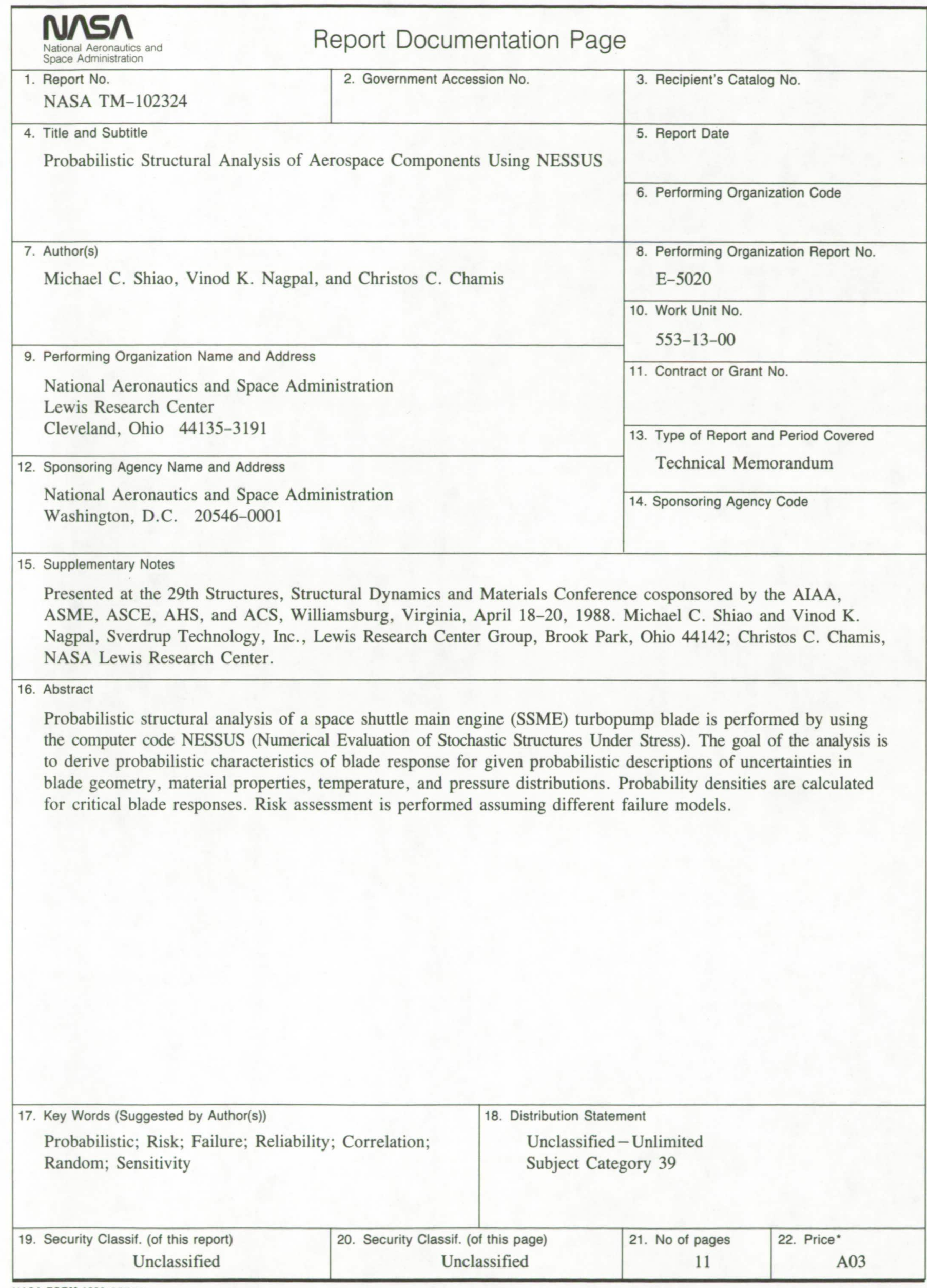


National Aeronautics and

Space Administration

Lewis Research Center

Cleveland, Ohio 44135

Official Business

Penalty for Private Use $\$ 300$

\section{ADDRESS CORRECTION REQUESTED}

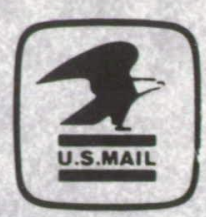

Postage and Fees Paid National Aeronautics and

Space Administration NASA. 451 